OPEN ACCESS

Edited by:

Paul Roesch,

University of Bayreuth, Germany

Reviewed by:

Li Ye,

Guangxi Medical University, China

Ming Sun,

Chinese Academy of Medical Sciences and Peking Union Medical College, China

*Correspondence: Yong Gao ygao387@ustc.edu.cn Zhirong Liu

zr@ahcdc.com.cn Jiajia Xie

xiejiajia@ustc.edu.cn

Specialty section: This article was submitted

to Viral Immunology,

a section of the journal

Frontiers in Immunology

Received: 12 April 2021

Accepted: 27 July 2021

Published: 17 August 2021

Citation:

Ding $C$, He J, Zhang $X$, Jiang $C$, Sun $Y$, Zhang Y, Chen Q, He H, Li W, Xie J,

Liu Z and Gao Y (2021) Crucial Mutations of Spike Protein on SARSCoV-2 Evolved to Variant Strains

Escaping Neutralization of Convalescent Plasmas and RBDSpecific Monoclonal Antibodies.

Front. Immunol. 12:693775. doi: 10.3389/fimmu.2021.693775

\section{Crucial Mutations of Spike Protein on SARS-CoV-2 Evolved to Variant Strains Escaping Neutralization of Convalescent Plasmas and RBD- Specific Monoclonal Antibodies}

\author{
Chengchao Ding ${ }^{1}$, Jun $\mathrm{He}^{2}$, Xiangyu Zhang ${ }^{1}$, Chengcheng Jiang ${ }^{1}$, Yong Sun ${ }^{2}$, \\ Yuqing Zhang ${ }^{1}$, Qingqing Chen ${ }^{2}$, Hongliang He ${ }^{1}$, Wenting $\mathrm{Li}^{1}$, Jiajia Xie ${ }^{1 *}$, Zhirong $\mathrm{Liu}^{2 *}$ \\ and Yong Gao ${ }^{1 *}$ \\ ${ }^{1}$ The First Affiliated Hospital of USTC, Division of Life Sciences and Medicine, University of Science and Technology of China \\ (USTC), Hefei, China, ${ }^{2}$ Department of Microbiology, Anhui Provincial Center for Disease Control and Prevention, Hefei, China
}

Small number of SARS-CoV-2 epidemic lineages did not efficiently exhibit a neutralization profile, while single amino acid mutation in the spike protein has not been confirmed in altering viral antigenicity resulting in immune escape. To identify crucial mutations in spike protein that escape humoral immune response, we evaluated the cross-neutralization of convalescent plasmas and RBD-specific monoclonal antibodies (mAbs) against various spike protein-based pseudoviruses. Three of 24 SARS-CoV-2 pseudoviruses containing different mutations in spike protein, including D614G, A475V, and E484Q, consistently showed an altered sensitivity to neutralization by convalescent plasmas. A475V and E484Q mutants are highly resistant to neutralization by mAb B38 and 2-4, suggesting that some crucial mutations in spike protein might evolve SARS-CoV-2 variants capable of escaping humoral immune response.

Keywords: SARS-CoV-2, crucial mutation, cross-neutralization, monoclonal antibody, spike protein

\section{INTRODUCTION}

Since the coronavirus disease 2019 (COVID-19) pandemic is still spreading globally, specific antibody therapy and vaccine have now been approved to combat the causative agent SARS-CoV-2 $(1,2)$. Direct transfusion of specific SARS-CoV-2 antibodies, i.e., passive immunization, allows the individual to acquire immediate but short immunity, while the COVID-19 vaccine is able to elicit long-lasting immunity in the vaccinees. Both of the two strategies have showed a remarkable effect on the control of the epidemic and/or the recovery of the patients $(3,4)$.

Different from other RNA viruses, the non-structural protein nsp14 of SARS-CoV-2 has 3'-5' exonuclease activity correcting the nucleotide mismatches occurred in the process of viral genome transcription $(5,6)$. Therefore, the overall mutation frequency of SARS-CoV-2 genome is lower than that of HIV-1 and influenza viruses. However, the recent emergence of a new circulating mutant strain has raised public concern about the protection efficiency of the COVID-19 vaccine and antibody therapy. Single amino acid mutation in the spike protein could affect the 
physicochemical property and structure of the protein, which subsequently alters the affinity of the receptor binding domain (RBD) with the viral receptor, human angiotensin-converting enzyme 2 (hACE2) $(7,8)$. Importantly, the emergence of these amino acid mutations might result in a reduced neutralization ability of the immune serum from vaccinees or the existing antibodies in recovered COVID-19 patients, causing a new wave of the epidemic.

The mutation in the spike protein not only affects the replication capacity and infectivity of the virus, but also impacts the host immune response. During the SARS epidemic in 2003, the single amino acid mutation D480A/G in the RBD domain of SARS-CoV spike protein gradually became dominant, and subsequent study confirmed that this mutation occurred in a critical site and enabled the mutant escaping neutralizing antibodies (9). In contrast, early in the COVID-19 pandemic, SARS-CoV-2 spike mutation D614G rapidly became globally dominant. Several studies demonstrated that the D614G mutation enhanced the replication of the mutated virus in the lung epithelial cells (10). This variant has a higher infectiousness due to its higher viral load in the respiratory secretions. However, a recent study indicated that the neutralizing activity of immune sera from vaccinees against G614 variant was better than the original one, and revealed that the structure of the G614 spike had a more open ACE2 binding site in the RBD region (11). In addition, several recent studies have focused on the neutralizing activity of vaccine-elicited humoral immunity against new circulating mutant lineages, including B.1.1.7 (United Kingdom, bearing mutations 69-70 del, 144 del, N501Y, A570D, D614G, P681H, T716I, S982A, and D1118H in the spike protein), B.1.429 (United States, bearing mutations S13I, W152C, L452R, and D614G in the spike protein), B.1.351(South Africa, bearing mutations D80A, D215G, K417N, E484K, N501Y D614G, and A701V in the spike protein), and P.1 and P.2 (Brazil, bearing certain mutations E484K, D614G, and V1176F, etc. in the spike protein) $(12,13)$. Amongst these mutants, the neutralization susceptibility of B.1.351 was significantly decreased to the immune sera from vaccinees (14-16). However, crucial single amino acid mutations that altered the antigenicity of the spike protein have not been fully explored, since natural variants generally containing multiple mutations at different sites.

Although the mutation frequency of SARS-CoV-2 is lower than that of other RNA viruses without a correction mechanism, the rapid global spread of SARS-CoV-2 and large amounts of infections still produce sufficient natural mutations for the virus. Spike protein is the main antigen that induces protective immune responses, thus, become the main target of neutralizing antibodies and currently used vaccines $(17,18)$. Antigenic drift may occur in the glycoprotein of the mutant virus, thus, antibodies derived from the original strain might only afford a partial cross-neutralizing effect against different variants. Obviously, it is important to keep a close eye on the evolution and mutation of the spike protein during transmission of the virus. To identify the crucial amino acid mutations that alter the antigenicity of the virus, we generated 24 pseudoviruses with different individual spike mutations that occurred naturally and frequently in the epidemic to determine their possible resistance to specific immune responses, and found that two variants with a crucial single amino acid mutation on the spike protein were capable of escaping the neutralization by convalescent plasma and RBD-specific monoclonal antibodies.

\section{METHODS}

\section{Human Samples}

A total of 20 recovered COVID-19 patients from the First Affiliated Hospital of USTC were enrolled in this study. All participants provided written informed consent. The convalescent plasma samples were obtained at 3-5 months after the initiation of the disease.

\section{Construction of Plasmids and Cell Lines}

SARS-CoV-2 surface glycoprotein gene (GenBank: MN_908947) with C-terminal 19 amino acids deletion was codon-optimized for Homo sapiens and cloned into the eukaryotic expression plasmid pcDNA3.1(+) between the Hind III and BamH I sites to generate the envelope expression plasmid pcDNA3.1(+)-Opt-S. The lentiviral packaging plasmid pNL4-3 Luc+R-E- carrying an Env-defective and luciferase-expressed HIV-1 genome was generously gifted by Binlian Sun, Jianghan University. To simulate the virus entry assay in vitro, HEK293T cell line exogenously expressing hACE2 (HEK293T/hACE2) was constructed for pseudovirus neutralization assay in this study. HEK $293 \mathrm{~F}$ cell line was generously gifted by Tengchuan Jin, University of Science and Technology of China. HEK293T and HEK293T/hACE2 cells were maintained in high glucose Dulbecco's Modified Eagle Medium (Gibco) supplemented with $10 \%$ FBS (ExCell), penicillin $(100 \mathrm{IU} / \mathrm{ml})$, and streptomycin $(100 \mu \mathrm{g} / \mathrm{ml})$ in a $5 \% \mathrm{CO}_{2}$ environment at $37^{\circ} \mathrm{C}$ and passaged every 3 days. HEK293F cells were maintained in Chemically Defined Medium (Union-Biotech Co. Ltd, Shanghai) supplemented with penicillin $(100 \mathrm{IU} / \mathrm{ml})$ and streptomycin $(100$ $\mu \mathrm{g} / \mathrm{ml}$ ) in $37^{\circ} \mathrm{C}$ shaker at $120 \mathrm{rpm}$ with $5 \% \mathrm{CO}_{2}$.

\section{Selection of Spike Mutation and Production of Corresponding Pseudoviruses}

For the selection of spike mutations that occurred naturally and frequently, we retrieved a total of 24 sequences of $\mathrm{S}$ gene in the $\mathrm{RBD}$ and hACE2 interaction region ( $\mathrm{RBD} / \mathrm{hACE} 2, \mathrm{n}=8), \mathrm{RBD}$ region but no interaction with hACE2 (RBD/Non-hACE2, $\mathrm{n}=$ 10 ), and Non-RBD region (Non-RBD, $n=6$ ) in the GISAID database up to March 1, 2021. All selected single amino acid mutations in the above-mentioned regions are shown in Figure 1. To generate the plasmids encoding various spike mutants, pcDNA3.1(+)-Opt-S plasmid was used as a template to perform site-directed mutagenesis through recombinational PCR amplification. The resultant PCR product was then digested using DpnI restriction endonuclease to exclude the template 


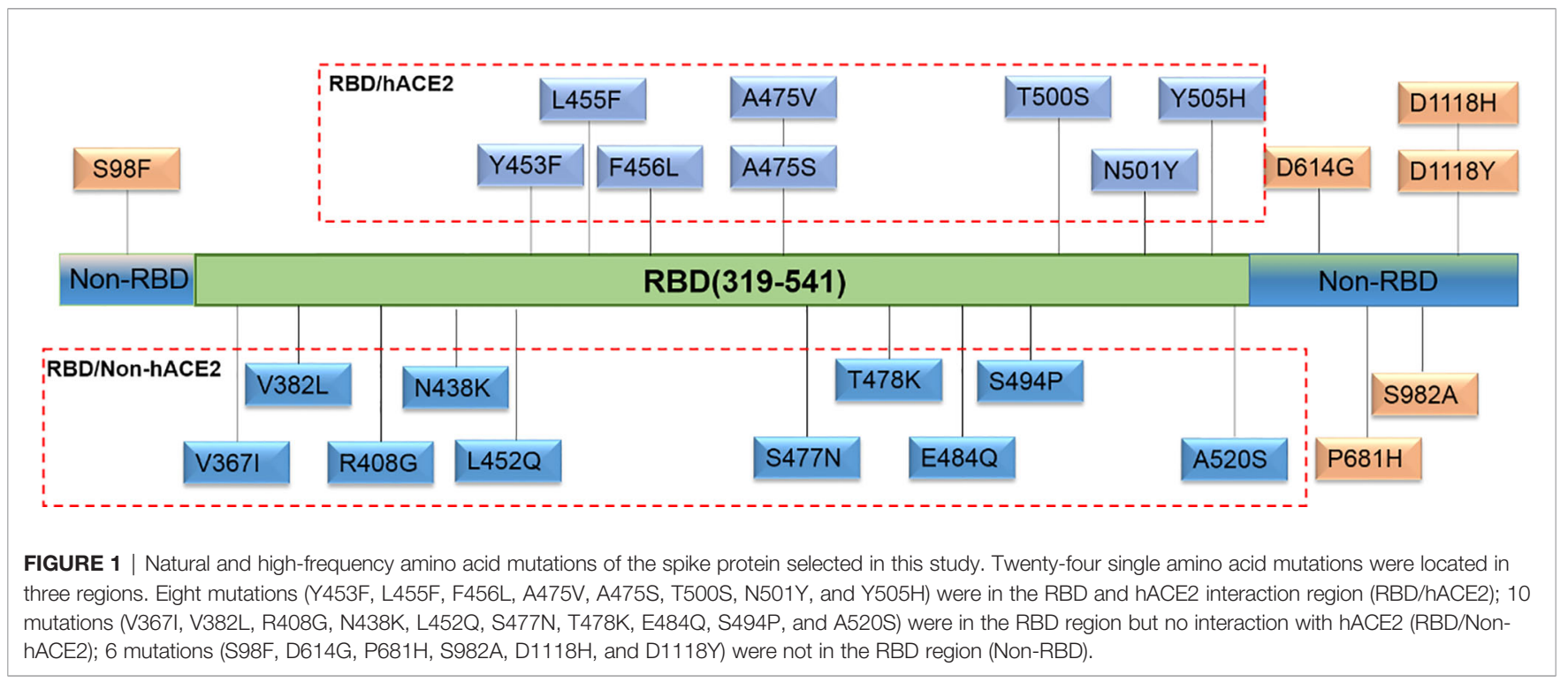

plasmid. The digested product was transformed into Stbl3 competent cells, and single clones were selected and sequenced.

Pseudoviruses with spike mutations were produced by the cotransfection of HEK293T cells with pNL4-3 Luc+R-E- and plasmids encoding various spike mutations, while Wuhan reference pseudovirus was produced with pNL4-3 Luc+R-Eand pcDNA3.1(+)-Opt-S. Briefly, 24 hours prior to transfection, HEK293T cells were prepared at $7 \times 10^{6}$ cells $/ \mathrm{T}_{75}$ and cultured at $37^{\circ} \mathrm{C}$ with $5 \% \mathrm{CO}_{2}$. The two plasmids $(50 \mathrm{mg}, 1: 1$ copy numbers) were then transfected into the HEK293T cells using polyetherimide (PEI). The supernatants were harvested at $48 \mathrm{~h}$ post transfection, centrifuged at $300 \times \mathrm{g}$ for $10 \mathrm{~min}$, passed through $0.45 \mu \mathrm{m}$ filter, and stored at $-80^{\circ} \mathrm{C}$ in $1 \mathrm{~mL}$ aliquots until use.

\section{Expression and Purification of RBD-Specific mAbs}

The amino acid sequences of three published SARS-CoV-2 mAbs (mAb CC12.1, mAb 2-4, and mAb B38) were downloaded from the PDB (protein data bank) database (http://www.rcsb.org/). Each antibody DNA sequence transformed from the amino acid sequence was codonoptimized for human cells. The resultant sequences of paired heavy and light chain variable regions were then synthesized (General Biosystems Co. Ltd, Anhui, China) and separately cloned into expression vectors containing human IgG1 constant regions which were co-transfected into HEK293F cells using PEI and cultured in a $37^{\circ} \mathrm{C}$ shaker at $120 \mathrm{rpm}$ with $5 \% \mathrm{CO}_{2}$. The culture supernatant was collected 5 days post-transfection and the produced mAbs were purified using the Protein A sepharose column (GE Healthcare) and eluted with buffer containing $20 \mathrm{mM} \mathrm{Na}_{2} \mathrm{HPO}_{4}, 150 \mathrm{mM} \mathrm{NaCl}$, and substitution with PBS by centrifugation with $10 \mathrm{kDa}$ molecular weight cut-off membrane centrifugal filter (Millipore).

\section{Pseudovirus Neutralization Assay}

Pseudovirus neutralization assay was performed by incubating the pseudovirus with serial dilutions of convalescent plasma or purified mAbs and detecting the luciferase expression level in target cells. Briefly, HEK293T/hACE2 cells were seeded in 96well cell plates at 10,000 cells/well in the culture medium $24 \mathrm{~h}$ prior to detection. The convalescent plasmas were 2-fold serially diluted in the culture medium with the initial dilution of 1:20 (dilution range of 1:20-1:2,560). Purified mAbs were 5-fold serially diluted in the culture medium with the initial dilution of $10 \mu \mathrm{g} / \mathrm{mL}$ (concentration range of $10 \mu \mathrm{g} / \mathrm{mL}-1.28 \times 10^{-4} \mu \mathrm{g} /$ $\mathrm{mL}$ ). A total of $50 \mu \mathrm{L}$ of pseudovirus with the values of relative luminescence unit (RLU) at approximately $1.0 \times 10^{5}$ was incubated with diluted plasma or $\mathrm{mAb}$ at $37^{\circ} \mathrm{C}$ for $1 \mathrm{~h}$, which were then added to HEK293T/hACE2 cells. After $48 \mathrm{~h}$ of incubation at $37^{\circ} \mathrm{C}$ with $5 \% \mathrm{CO}_{2}$, culture supernatants were removed and the values of RLU were measured by the Britelite plus Reporter Gene Assay System (PerkinElmer). The illustration of pseudovirus production and neutralization assay is shown in Figure 2.

\section{Statistical Analysis}

The NTIC50 or IC50 was defined as the dilution (plasma dilution to NTIC50, mAb concentration to IC50) at which the RLU values were reduced by $50 \%$ compared with the pseudovirus control wells calculated by the GraphPad Prism 8 (GraphPad Software). The difference of geometric mean titer (GMT) in two independent groups was tested for statistical significance with a Mann-Whitney U test in the GraphPad Prism 8. The difference of GMT in two paired groups was tested for statistical significance with a Wilcoxon matched-pairs signed rank test in the GraphPad Prism 8. Differences were considered statistically significant at ${ }^{\star} p<0.05,{ }^{* *} p<0.01$, or ${ }^{* *} p<0.001$. 

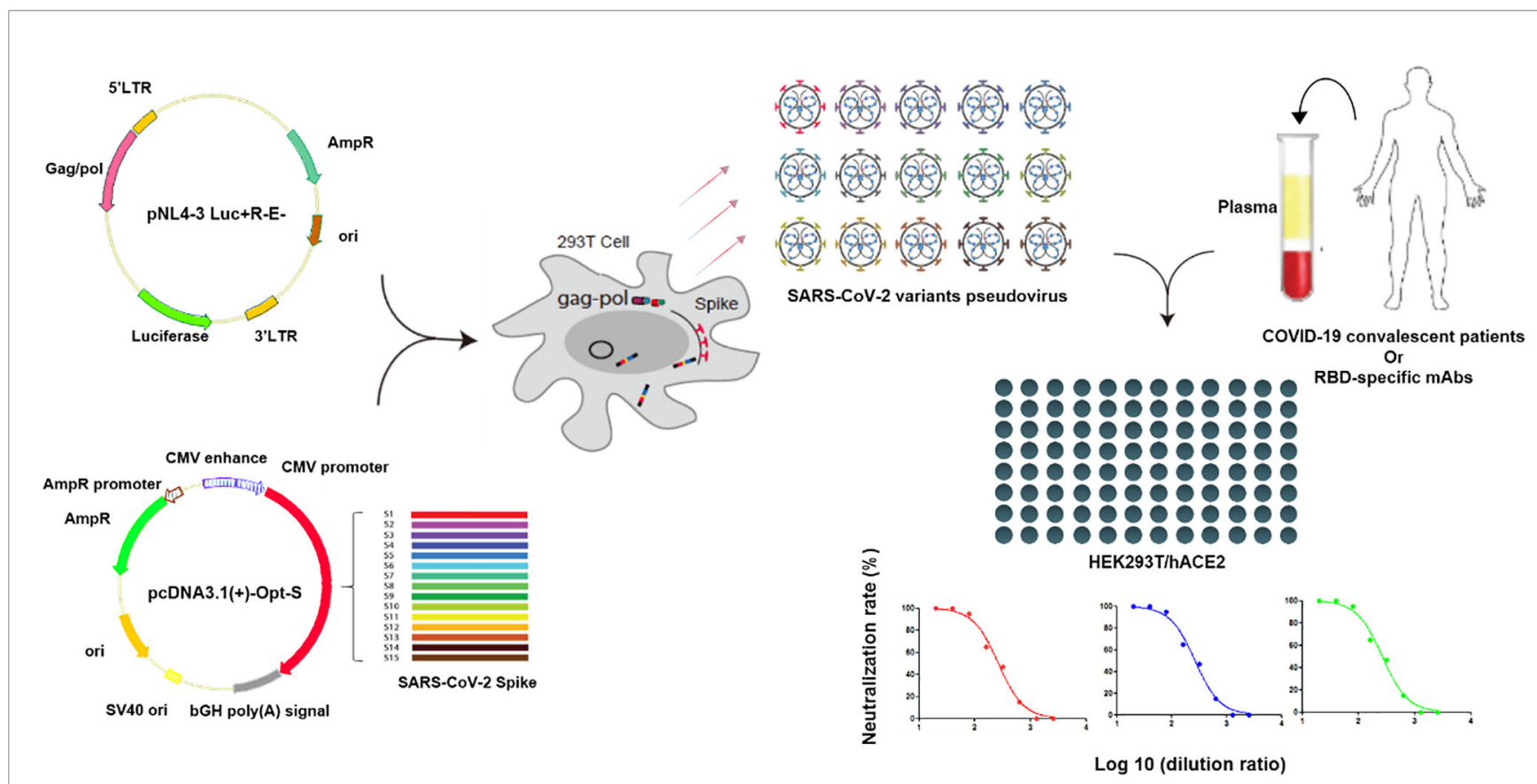

FIGURE 2 | Illustration of pseudovirus production and neutralization assay. Pseudoviruses were produced by the co-transfection of HEK293T cells with pNL4-3 Luc+R-E- and pcDNA3.1(+)-Opt-S plasmids expressing spike proteins. Neutralization assay was performed by incubating the pseudovirus with diluted convalescent plasmas or mAbs, and the neutralization rates were detected in the HEK293T/hACE2 cells.

\section{RESULTS}

\section{Neutralizing Antibody Responses Against the Wuhan Reference Pseudovirus in Convalescent Plasma}

To assess the neutralizing antibody responses against the Wuhan reference pseudovirus in the plasmas from COVID-19 recovered patients, we included 20 patients from the First Affiliated Hospital of USTC. The time between the recovery to sample collection was 3-5 months. As shown in Figure 3A, NTIC50 of 20 convalescent plasma samples ranged from 1.5 to 3.0 of $\log 10$, with a GMT of neutralizing antibodies 110.2 (95\% CI 64.4188.7). As shown in Figure 3C, the neutralization curves of five samples $(2 \#, 5 \#, 7 \#, 8 \#$, and 12\#) show a significantly higher GMT (579.4, 95\% CI 353.0-950.9, Figure 3B left) than others (63.4, 95\% CI 44.0-91.3, Figure 3B right) against the Wuhan reference pseudovirus. We deduced that convalescent plasma with a higher GMT might bear more abundant neutralizing antibodies against SARS-CoV-2. Thus, five plasma samples with a higher GMT were then used to explore the impact on humoral immune response against pseudovirus bearing the various mutated spike proteins.

\section{Neutralizing Antibody Responses Against Variant Pseudoviruses in Five High GMT Convalescent Plasma Samples}

To explore the difference of the neutralizing antibody responses against variant pseudoviruses, we constructed a total 24
SARS-CoV-2 spike protein pseudoviruses with a single amino acid mutation in RBD/hACE2 $(\mathrm{n}=8)$, RBD/Non-hACE2 $(\mathrm{n}=10)$, or Non-RBD $(\mathrm{n}=6)$ regions. All selected single amino acid mutations in the above-mentioned regions are shown in Figure 1. Since five plasma samples showed a higher GMT in the convalescent plasma neutralization assay, we next determined how these plasma samples reacted to the variant pseudoviruses. The illustration of pseudovirus production and neutralization assay is shown in Figure 2. The neutralization curves of the five samples $(2 \#, 5 \#, 7 \#, 8 \#$, and 12\#) against variant pseudoviruses are shown in Supplementary Figure 1. When the GMT values of variants with mutations in each region were pooled together to separately analyze the neutralizing activity in the five samples, no significant differences were observed (shown in Figure 4). However, the GMT of the Non-RBD group was slightly higher than that in other two groups (i.e., RBD/Non-hACE2 and Non-RBD).

\section{Relative Neutralization Activity of Variant Pseudoviruses}

Antigenic drift may occur in the glycoprotein of mutant viruses in the global pandemic in a short time. To investigate the immune escape of variants that occurred naturally, we analyzed the NTIC50 of five high GMT convalescent plasma samples against 24 variant pseudoviruses. NTIC50 of variant pseudoviruses relative to the Wuhan reference pseudovirus are shown in Figure 5A. Three variant pseudoviruses with a single amino acid mutation including A475V, E484Q, and D614G 
A

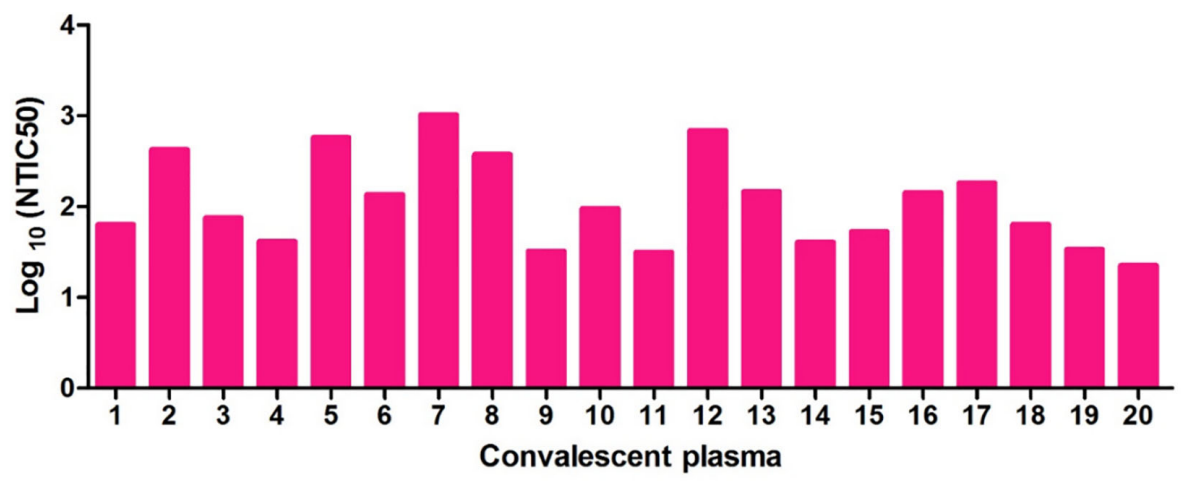

B

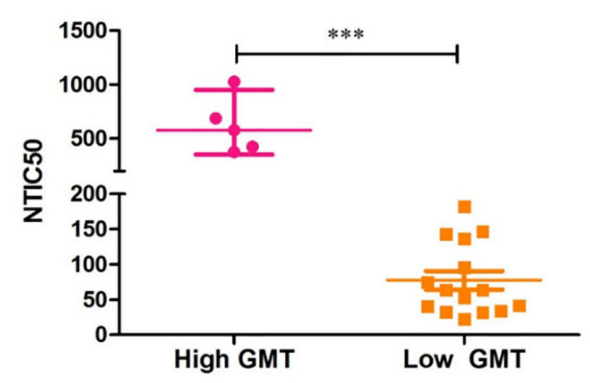

C

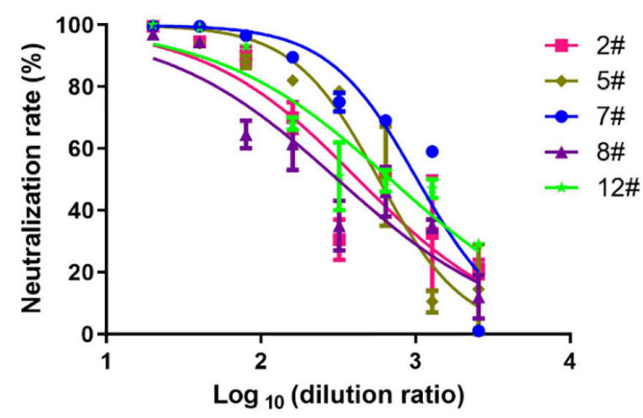

FIGURE 3 | Neutralization titers of convalescent plasmas against pseudovirus bearing the Wuhan reference spike protein. (A) Neutralization titer of $50 \%$ Wuhan pseudovirus inhibition (NTIC50) of 20 plasma samples from convalescent COVID-19 patients. (B) Clustering analysis in higher and lower GMT convalescent plasma samples. The difference of GMT in two groups was tested for statistical significance with a Mann-Whitney U test in the GraphPad Prism 8. Differences were considered statistically significant at ${ }^{\star \star *} p<0.001$. (C) Neutralization curves of the five convalescent plasma samples (2\#, 5\#, 7\#, 8\#, and 12\#) that show a higher neutralization titer than others against the Wuhan pseudovirus.

consistently altered the neutralization sensitivity to the five convalescent plasma samples. When compared with the Wuhan reference pseudovirus $(\mathrm{GMT}=579.4)$, paired neutralization analysis showed that the convalescent plasma reduced the neutralizing activity against $\mathrm{A} 475 \mathrm{~V}$ (GMT $=341.5$, Figure 5B) and E484Q (GMT = 405.6, Figure 5C) variants but increased the neutralizing activity against the D614G variant $(\mathrm{GMT}=2,017.0$, Figure 5D). However, when the GMT values of these three variant pseudoviruses were analyzed for the relative neutralizing activity to the Wuhan reference pseudovirus, no significant differences were observed (Wuhan reference vs. A475V, $p=0.0625$; Wuhan reference vs. E484Q, $p=0.1250$; and Wuhan reference vs. D614G, $p=0.0625)$.

\section{Neutralization Analysis of RBD-Specific mAbs Against A475V and E484Q Variant Pseudoviruses}

Previous study indicated that the plasma from convalescent COVID-19 patients was rich in SARS-CoV-2 specific polyclonal antibodies (19). The results of the relative neutralizing activity analysis suggested that A475V and E484Q might decrease the sensitivity of the variant to the polyclonal antibodies from the convalescent plasma. Therefore, we finally explored whether these mutations impact the neutralizing activity of RBD-specific mAbs. The neutralization curves of three RBD-specific mAbs (CC12.1, 2-4, and B38) against the Wuhan reference pseudovirus, $\mathrm{A} 475 \mathrm{~V}$ and $\mathrm{E} 484 \mathrm{Q}$ variant pseudoviruses are respectively shown in Figures $\mathbf{6 A - C}$, respectively. The neutralizing activity against two variant pseudoviruses relative to the Wuhan reference pseudovirus is shown in Figures 6D, E. We found that CC12.1 was effective in neutralizing both $\mathrm{A} 475 \mathrm{~V}$ and $\mathrm{E} 484 \mathrm{Q}$ variant pseudoviruses. It is notable that antibody B38 was unable to effectively neutralize A475V variant pseudovirus (Figure 6D) while the neutralizing activity of antibody 2-4 against E484Q variant pseudovirus was also decreased nearly 1,000-fold (Figure 6E).

\section{DISCUSSION}

In the convalescent plasma, we speculated that samples with a high neutralization titer might contain more abundant specific neutralizing antibodies, and novel high-affinity neutralizing 
2\#

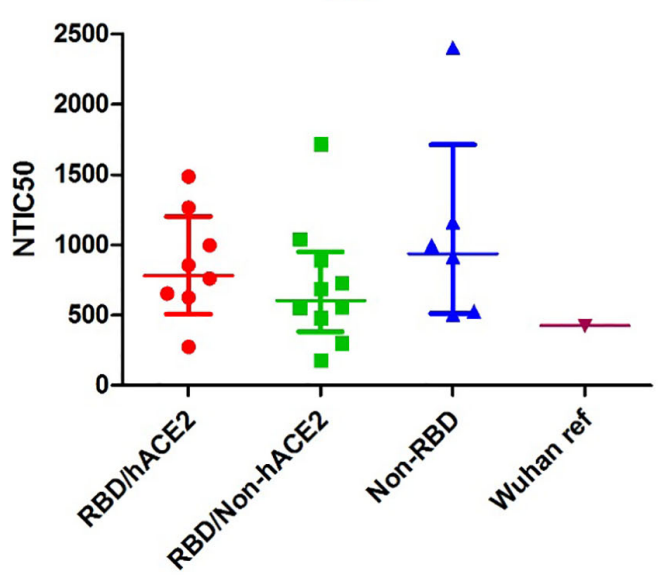

7\#

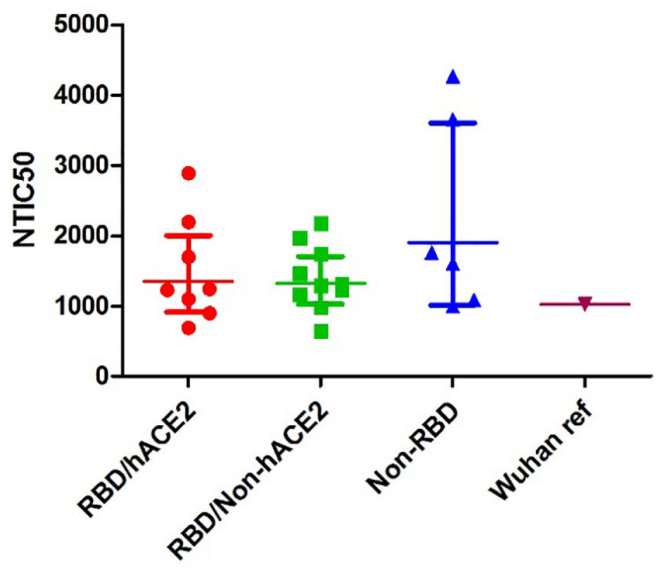

5\#

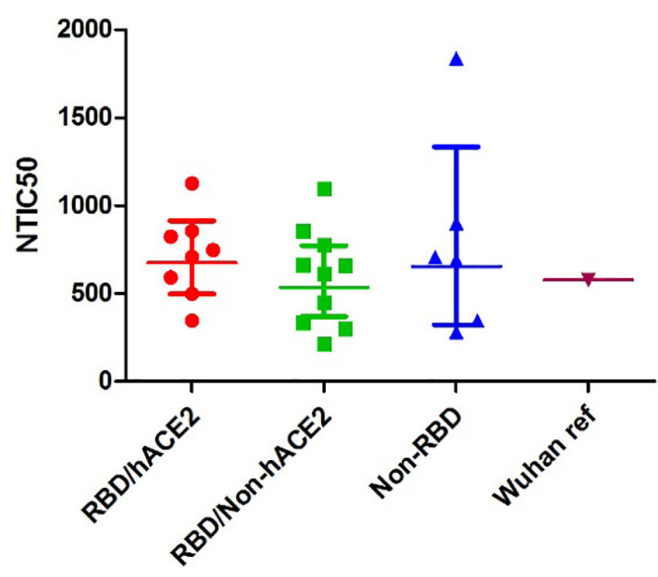

8\#

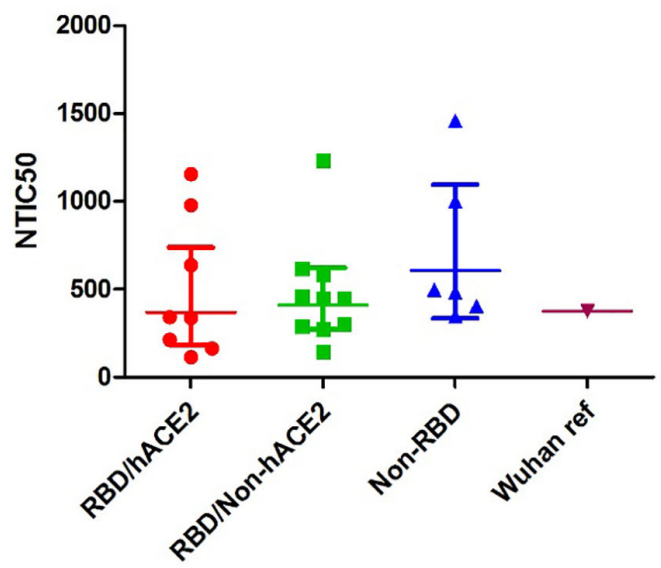

12\#

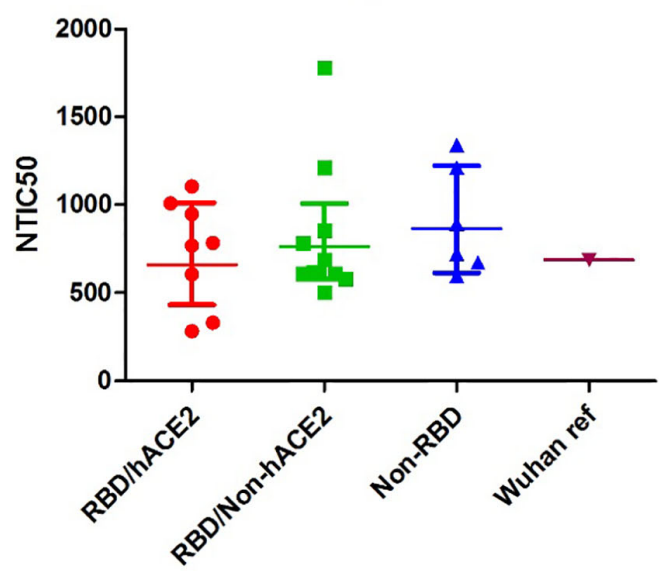

FIGURE 4 | NTIC50 against pseudovirus bearing the various spike mutants for the five convalescent plasma samples (2\#, 5\#, 7\#, 8\#, and 12\#) that show a higher neutralization titer against the Wuhan reference pseudovirus. The difference of GMT in different regions was tested for statistical significance with a Mann-Whitney $U$ test in the GraphPad Prism 8. 


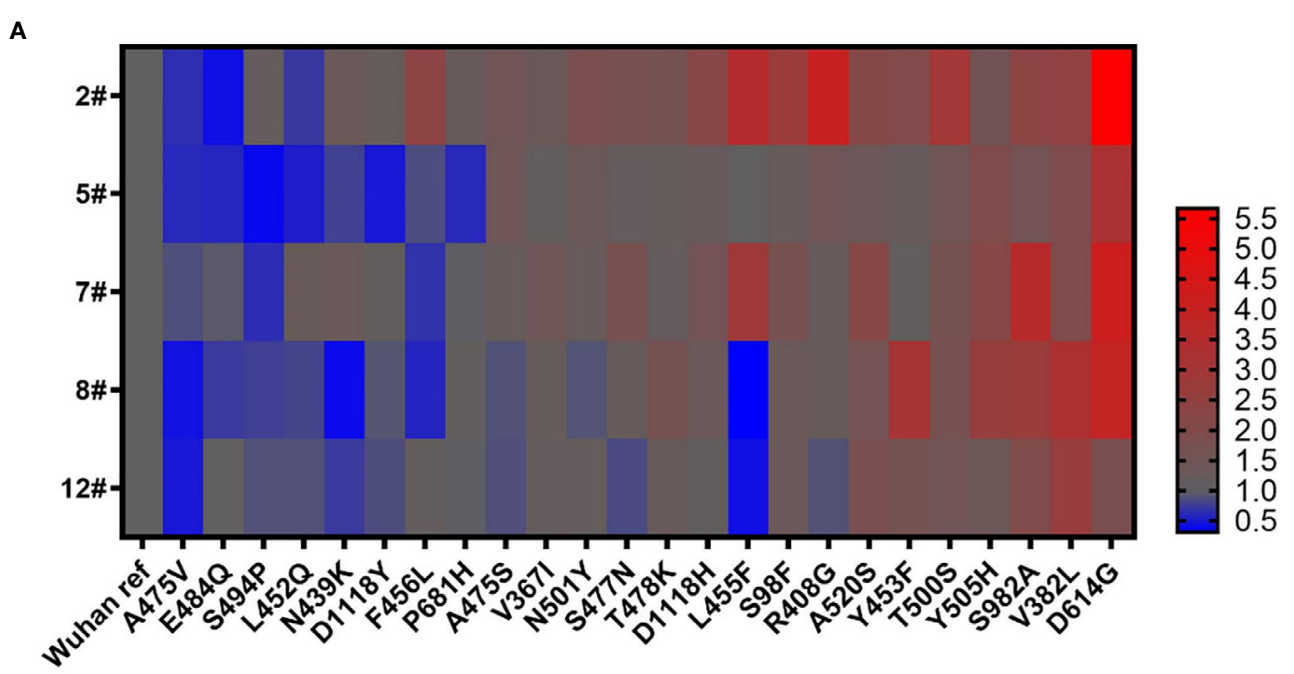

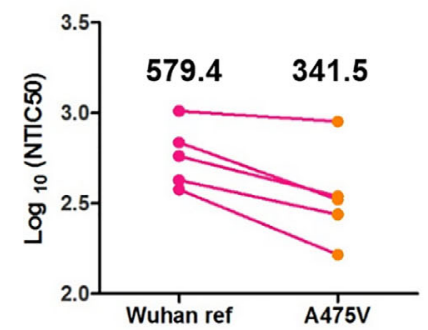

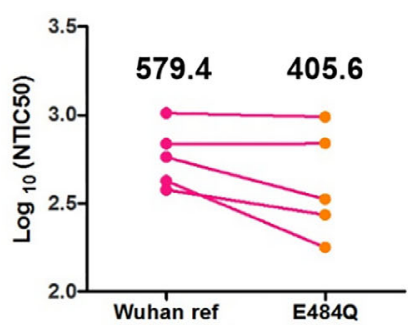

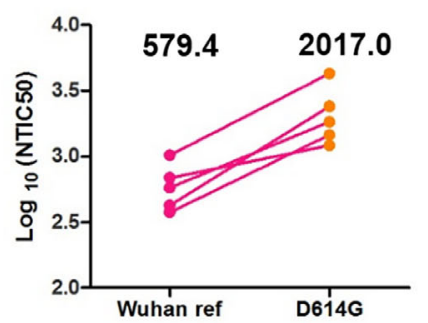

FIGURE 5 | Neutralization analysis of convalescent plasmas $(n=5)$ against the Wuhan reference and variant pseudoviruses. (A) Heat map of the relative neutralizing activities of the five convalescent plasma samples against pseudoviruses bearing the variant spike proteins compared to the Wuhan reference pseudovirus. (Blue denotes reduction; red denotes increase). Paired neutralization analyses of five convalescent plasma samples against the Wuhan reference and variant pseudoviruses of A475V, E484Q, and D614G are shown in (B-D), respectively. GMT is shown above each graph. The difference of GMT was tested for statistical significance with a Wilcoxon matched-pairs signed rank test in the GraphPad Prism 8.

antibodies or broadly neutralizing antibodies against SARS-CoV-2 variants are more likely to emerge in these individuals. Therefore, in the present study, we first used the plasma samples with a high neutralization titer to explore the cross-neutralization against the variants with different mutations in the spike protein. We found that there were slight differences of the neutralizing activity among the three groups (RBD/hACE2, RBD/Non-hACE2, and Non-RBD) of spike mutants but were insignificant (Figure 4). It was accepted that the mutations located in the $\mathrm{RBD} / \mathrm{hACE} 2$ region more likely alter the viral neutralization reactivity to antibodies (20). Our results of the neutralization of the convalescent plasma against different spike variants indicated that mutations in either RBD/hACE2 or $\mathrm{RBD} /$ Non-hACE2 interaction regions slightly decreased the neutralizing activity of the convalescent plasma against the corresponding pseudoviruses. The reason might lie in the fact that there are multiple neutralizing antibodies in the patients and a few mutations in the virus might not sufficiently enable the virus escape from immune attack. On the other hand, since the mutation in the non-RBD and ACE2 interaction region resulted in a decreased neutralization sensitivity, we should also pay attention to the mutations in the entire spike protein region in order to fully monitor the possible mutations causing immune escape.

In paired neutralization analysis of the Wuhan reference and D614G variant pseudoviruses, we found that the D614G variant pseudovirus was more sensitive to neutralization by the convalescent plasma (Figure 5). Previous study revealed that the percentage of RBD "up" conformation in the G614 spike was higher than that in the D614 spike (21), which might explain that the D614G variant is more infectious while more sensitive to neutralization. Therefore, we suspect that D614G and possibly other sensitive variants might not be challenges for current COVID-19 vaccines. However, we also found that certain variants with a single amino acid mutation (e.g., A475V and E484Q) might be able to escape neutralization by plasma from COVID-19 recovered patients. The decreased neutralization activities highlight that we should absolutely focus on the effect of vaccines against these variants that are resistant to humoral immunity, especially when these mutations occur simultaneously. Additionally, we speculate that the decreased neutralization activities of A475V and E484Q might be due to their resistance to some mAbs. The plasma neutralization assay indicated that epidemic variants 
A

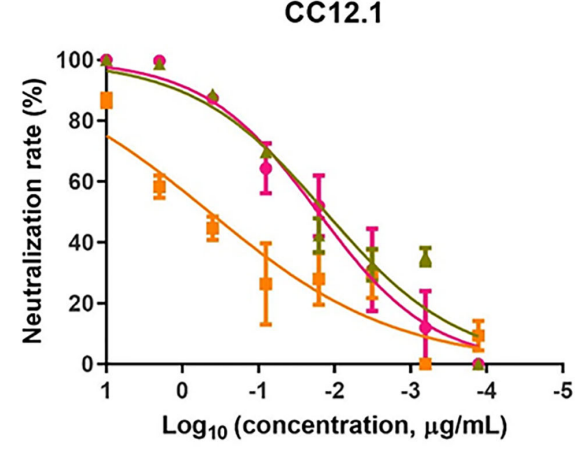

B

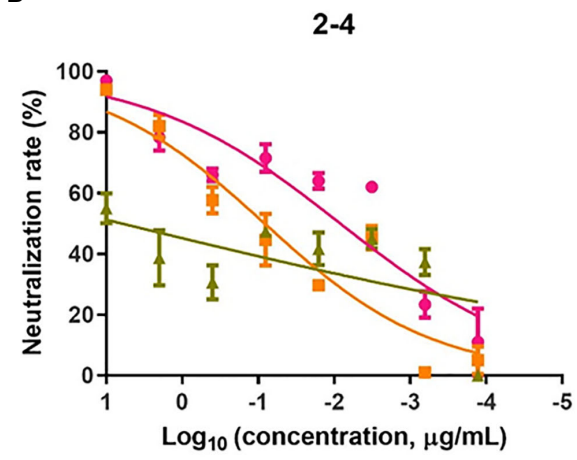

C

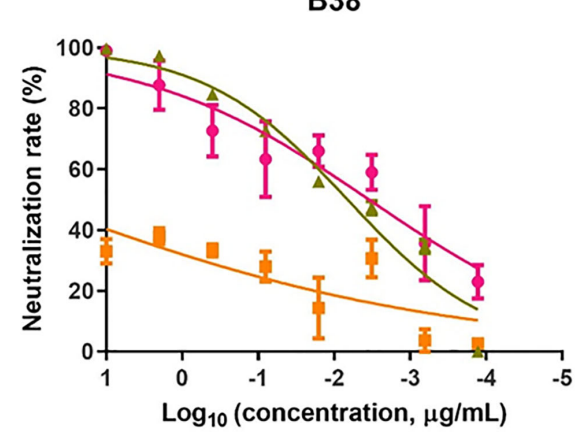

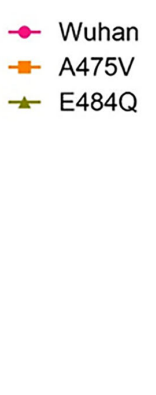

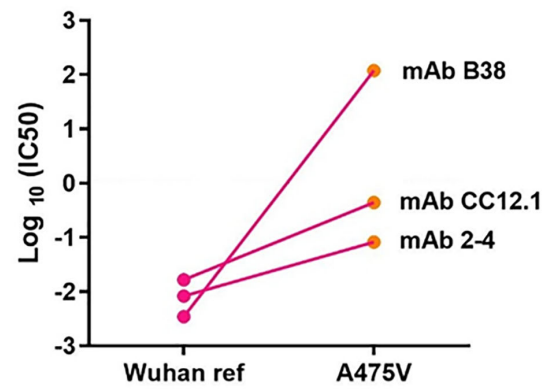

E

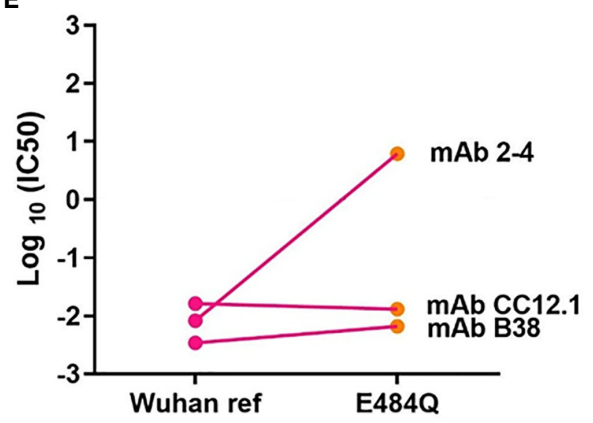

FIGURE 6 | Neutralization analysis of RBD-specific mAbs $(n=3)$ against pseudoviruses with the Wuhan reference spike protein and variant spike protein A475V or E484Q. Neutralization curves of three mAbs (CC12.1, (A); 2-4, (B); B38, (C) against the Wuhan reference pseudovirus, A475V variant pseudovirus, and E484Q variant pseudovirus in the HEK293T/hACE2 cells. Paired neutralization analyses of three mAbs against the Wuhan reference and variant pseudoviruses of A475V and E484Q are shown in (D, E). The 50\% pseudovirus inhibition concentration (IC50) of mAb was defined as the concentration at which the RLU values were reduced by $50 \%$ compared with the pseudovirus control wells, which was calculated in the GraphPad Prism 8 (GraphPad Software).

including either of the mutant sites described above might indeed partially escape neutralization by humoral immunity.

Importantly, the aforementioned immune escape was further confirmed through the neutralization assay with RBD-specific mAbs. The neutralizing activity of mAb B38 against A475V mutant was reduced 10,000 folds when compared with the wild type virus (Figure 6), while the efficacy of mAb 2-4 in neutralizing the E484Q mutant also decreased up to 1,000 folds (Figure 6). We found that A475 is one of the direct interaction residues between antibody and RBD when reviewing the structural basis of
mAb B38 in the original study (22). Similarly, a previous cryo-EM analysis of $\mathrm{mAb} 2-4$ in complex with the spike trimer shows that E484 is also one of key sites binding to this specific antibody (23). The phenomenon of a decreased neutralization activity of some RBD-specific mAbs against RBD mutants suggests that cocktail therapy with multiple mAbs might be necessary in order to effectively suppress SARS-CoV-2 replication and to avoid immune escape. A potential limitation of this study lies in the lack of neutralization activity analysis with vaccine-induced plasma. However, a previous study indicated that neutralization 
activities consist between vaccination and convalescent sera (24). It should be noted that, compared with antibody therapy, it is much more comprehensive to develop a COVID-19 vaccine that can elicit broadly neutralizing antibodies against various variants other than specific ones against a few strains.

\section{DATA AVAILABILITY STATEMENT}

The raw data supporting the conclusions of this article will be made available by the authors, without undue reservation.

\section{ETHICS STATEMENT}

The studies involving human participants were reviewed and approved by the ethics committee of the First Affiliated Hospital of USTC. The patients/participants provided their written informed consent to participate in this study.

\section{AUTHOR CONTRIBUTIONS}

JX, ZL, and YG designed the study. CD, JH, XZ, CJ, YS, YZ, QC, $\mathrm{WL}$, and $\mathrm{HH}$ performed the experiments. $\mathrm{CD}$ analyzed the data. $\mathrm{CD}$ and YG wrote the manuscript. All authors contributed to the article and approved the submitted version.

\section{REFERENCES}

1. Ramasamy MN, Minassian AM, Ewer KJ, Flaxman AL, Folegatti PM, Owens DR, et al. Safety and Immunogenicity of ChAdOx1 Ncov-19 Vaccine Administered in a Prime-Boost Regimen in Young and Old Adults (COV002): A Single-Blind, Randomised, Controlled, Phase 2/3 Trial. Lancet (2020) 396:1979-93. doi: 10.1016/S0140-6736(20)32466-1

2. Weinreich DM, Sivapalasingam S, Norton T, Ali S, Gao H, Bhore R, et al. REGN-COV2, a Neutralizing Antibody Cocktail, in Outpatients With Covid19. N Engl J Med (2021) 384:238-51. doi: 10.1056/NEJMoa2035002

3. Baum A, Fulton BO, Wloga E, Copin R, Pascal KE, Russo V, et al. Antibody Cocktail to SARS-CoV-2 Spike Protein Prevents Rapid Mutational Escape Seen With Individual Antibodies. Science (2020) 369:1014-18. doi: 10.1126/ science.abd0831

4. Logunov DY, Dolzhikova IV, Shcheblyakov DV, Tukhvatulin AI, Zubkova OV, Dzharullaeva AS, et al. Safety and Efficacy of an Rad26 and Rad5 VectorBased Heterologous Prime-Boost COVID-19 Vaccine: An Interim Analysis of a Randomised Controlled Phase 3 Trial in Russia. Lancet (2021) 397:671-81. doi: 10.1016/S0140-6736(21)00234-8

5. Eckerle LD, Lu X, Sperry SM, Choi L, Denison MR. High Fidelity of Murine Hepatitis Virus Replication Is Decreased in Nsp14 Exoribonuclease Mutants. J Virol (2007) 81:12135-44. doi: 10.1128/JVI.01296-07

6. Ma Y, Wu L, Shaw N, Gao Y, Wang J, Sun Y, et al. Structural Basis and Functional Analysis of the SARS Coronavirus Nsp14-Nsp10 Complex. Proc Natl Acad Sci USA (2015) 112:9436-41. doi: 10.1073/pnas.1508686112

7. Luan B, Wang H, Huynh T. Molecular Mechanism of the N501Y Mutation for Enhanced Binding Between SARS-CoV-2's Spike Protein and Human ACE2 Receptor. FEBS Lett (2021) 595(10):1454-61. doi: 10.1101/2021.01.04.425316

8. Kemenesi G, Toth GE, Bajusz D, Keseru GM, Terhes G, Burian K, et al. Effect of An 84-Bp Deletion of the Receptor-Binding Domain on the ACE2 Binding

\section{FUNDING}

This work is supported by the Key Research and Development Project of Anhui Province (202104j07020042; 202104a07020032), Special Project for Emergency Scientific and Technological Research on New Coronavirus Infection (YD9110002001), Emergency Research Project of Novel Coronavirus Infection of Anhui Province (202004a07020002; 202004a07020004), Postdoctoral Research Foundation of China (2021M693076; 2020M670084ZX), the Fundamental Research Funds for the Central Universities (WK9110000166; WK9110000167), and the Hefei Comprehensive National Science Center.

\section{ACKNOWLEDGMENTS}

We thank Dr. Binlian Sun of Jianghan University for providing the plasmid pNL4-3 Luc+R-E-; and we also thank Dr. Tengchuan Jin from the University of Science and Technology of China for providing the HEK293F cells.

\section{SUPPLEMENTARY MATERIAL}

The Supplementary Material for this article can be found online at: https://www.frontiersin.org/articles/10.3389/fimmu.2021. 693775/full\#supplementary-material

Affinity of the SARS-CoV-2 Spike Protein: An In Silico Analysis. Genes (2021) 12:194. doi: 10.3390/genes12020194

9. Sui J, Aird DR, Tamin A, Murakami A, Yan M, Yammanuru A, et al. Broadening of Neutralization Activity to Directly Block a Dominant Antibody-Driven SARSCoronavirus Evolution Pathway. PloS Pathog (2008) 4:e1000197. doi: 10.1371/ journal.ppat.1000197

10. Shi P-Y, Plante J, Liu Y, Liu J, Xia H, Johnson B, et al. Spike Mutation D614G Alters SARS-CoV-2 Fitness and Neutralization Susceptibility. Res square (2020) 10:rs.3.rs-70482. doi: 10.21203/rs.3.rs-70482/v1

11. Yurkovetskiy L, Wang X, Pascal KE, Tomkins-Tinch C, Nyalile T, Wang Y, et al. : Structural and Functional Analysis of the D614G SARS-CoV-2 Spike Protein Variant. Cell (2020) 183:739-51.E8. doi: 10.1016/j.cell.2020.09.032

12. Shen X, Tang H, McDanal C, Wagh K, Fischer W, Theiler J, et al. SARS-CoV-2 Variant B.1.1.7 Is Susceptible to Neutralizing Antibodies Elicited by Ancestral Spike Vaccines. Cell Host Microbe (2021) 9(4):529-39. doi: 10.1101/2021.01. 27.428516

13. Long SW, Olsen RJ, Christensen PA, Subedi S, Olson R, Davis JJ, et al. Sequence Analysis of 20,453 Severe Acute Respiratory Syndrome Coronavirus 2 Genomes From the Houston Metropolitan Area Identifies the Emergence and Widespread Distribution of Multiple Isolates of All Major Variants of Concern. Am J Pathol (2021) 191(6):983-92. doi: 10.1101/2021.02.26. 21252227

14. Garcia-Beltran WF, Lam EC, St. Denis K, Nitido AD, Garcia ZH, Hauser BM, et al. Multiple SARS-CoV-2 Variants Escape Neutralization by VaccineInduced Humoral Immunity. Cell (2021) 184(9):2372-83. doi: 10.1101/ 2021.02.14.21251704

15. Hu J, Peng P, Wang K, Fang L, Luo F-y, Jin A-s, et al. Emerging SARS-CoV-2 Variants Reduce Neutralization Sensitivity to Convalescent Sera and Monoclonal Antibodies. Cell Mol Immunol (2021) 18:1061-3. doi: 10.1038/ s41423-021-00648-1 
16. Wibmer CK, Ayres F, Hermanus T, Madzivhandila M, Kgagudi P, Oosthuysen B, et al. SARS-CoV-2 501y.V2 Escapes Neutralization by South African COVID-19 Donor Plasma. Nat Med (2021) 27:9. doi: 10.1101/ 2021.01.18.427166

17. Su Q-D, Zou Y-N, Yi Y, Shen L-P, Ye X-Z, Zhang Y, et al. Recombinant SARSCoV-2 RBD With a Built in T Helper Epitope Induces Strong Neutralization Antibody Response. Vaccine (2021) 39:1241-7. doi: 10.1016/j.vaccine.2021.01.044

18. Srivastava V, Niu L, Phadke KS, Bellaire BH, Cho MW. Induction of Potent and Durable Neutralizing Antibodies Against SARS-CoV-2 Using a Receptor Binding Domain-Based Immunogen. Front Immunol (2021) 12:637982. doi: 10.3389/fimmu.2021.637982

19. Andreano E, Nicastri E, Paciello I, Pileri P, Manganaro N, Piccini G, et al. Extremely Potent Human Monoclonal Antibodies From COVID-19 Convalescent Patients. Cell (2021) 184:1821-35.e1816. doi: 10.1016/j.cell.2021.02.035

20. Brown EEF, Rezaei R, Jamieson TR, Dave J, Martin NT, Singaravelu R, et al. Characterization of Critical Determinants of ACE2-SARS CoV-2 RBD Interaction. Int J Mol Sci (2021) 22:2268. doi: 10.3390/ijms22052268

21. Weissman D, Alameh M-G, de Silva T, Collini P, Hornsby H, Brown R, et al. D614G Spike Mutation Increases SARS CoV-2 Susceptibility to Neutralization. Cell Host Microbe (2021) 29:23-31.e4. doi: 10.1016/j.chom. 2020.11.012

22. Wu Y, Wang F, Shen C, Peng W, Li D, Zhao C, et al. A Noncompeting Pair of Human Neutralizing Antibodies Block COVID-19 Virus Binding to Its Receptor ACE2. Science (2020) 368:1274-8. doi: 10.1126/science.abc2241
23. Liu L, Wang P, Nair MS, Yu J, Rapp M, Wang Q, et al. Potent Neutralizing Antibodies Against Multiple Epitopes on SARS-CoV-2 Spike. Nature (2020) 584:450-6. doi: 10.1038/s41586-020-2571-7

24. Supasa P, Zhou D, Dejnirattisai W, Liu C, Mentzer AJ, Ginn HM, et al. Reduced Neutralization of SARS-CoV-2 B.1.1.7 Variant by Convalescent and Vaccine Sera. Cell (2021) 184(8):2201-11. doi: 10.1016/j.cell.2021.02.033

Conflict of Interest: The authors declare that the research was conducted in the absence of any commercial or financial relationships that could be construed as a potential conflict of interest.

Publisher's Note: All claims expressed in this article are solely those of the authors and do not necessarily represent those of their affiliated organizations, or those of the publisher, the editors and the reviewers. Any product that may be evaluated in this article, or claim that may be made by its manufacturer, is not guaranteed or endorsed by the publisher.

Copyright (c) 2021 Ding, He, Zhang, Jiang, Sun, Zhang, Chen, He, Li, Xie, Liu and Gao. This is an open-access article distributed under the terms of the Creative Commons Attribution License (CC BY). The use, distribution or reproduction in other forums is permitted, provided the original author(s) and the copyright owner(s) are credited and that the original publication in this journal is cited, in accordance with accepted academic practice. No use, distribution or reproduction is permitted which does not comply with these terms. 